\title{
Die Bedeutung des tertiären Sektors und der öffentlichen Verwaltung in der Bundesstadt
}

\section{Einleitung}

Die Stadt Bern wird häufig als «Beamtenstadt» bezeichnet. In diesem Artikel sollen deshalb die Bedeutung der öffentlichen Verwaltung und der öffentlichen Hand allgemein als Arbeitgeber zahlenmäßig dargestellt und ihr Einfluß auf die Bevölkerungsund Wirtschaftsstruktur der Bundesstadt kurz aufgezeigt werden. Dabei können verschiedene Betrachtungsweisen angewandt werden:

- Die Stadt Bern als Wohnort oder als Arbeitsort.

- Der Anteil des Wirtschaftszweiges «Allgemeine öffentliche Verwaltung» oder der Anteil der Beschäftigten der öffentlichen Hand insgesamt.

- Die Beschäftigten bzw. Erwerbstätigen insgesamt oder nur die Vollbeschäftigten.

Im weiteren soll die Struktur der Stadt Bern auch mit derjenigen anderer Schweizer Städte verglichen werden.

Wie vielfach bei statistischen Vergleichen der Fall, stoßen wir auch in unserem Artikel auf definitorische Ungleichheiten (Begriff des tertiären Sektors, Definition der Erwerbstätigen/Beschäftigten, Erfassungsgrad derTeilzeitbeschäftigten u. a.), die im Rahmen des vorliegenden Artikels nur zum Teil bereinigt werden können. Im weiteren ist darauf hinzuweisen, daß bei derVolkszählung die Erwerbstätigen über die Wohnbevölkerung erfaßt werden (jede Person mit Angaben ihrer Erwerbstätigkeit bzw. Haupterwerbstätigkeit/Arbeitgeber). In der $\mathrm{Be}$ triebszählung dagegen kann eine Person bei mehreren Arbeitsstätten als Beschäftigter gezählt werden (z. B. Lehrer mit Teilpensum an mehreren Schulen).

Der Artikel erhält im heutigen Zeitpunkt zusätzliche Aktualität durch hängige politische Fragen wie die Abgeltung von externen Effekten durch die Region oder den Kanton an die Kernstadt und die geplante Verlegung von Bundesämtern aus der Stadt Bern. Im weiteren konnten erst seit kurzem verfügbare Daten in die Betrachtung einbezogen werden (Volkszählung 1980: Beschäftigte am Arbeitsort; Betriebszählung 1985).

\section{Begriffsdefinitionen}

Erwerbstätige (Volkszählung): Personen von 15 und mehr Jahren, die während wenigstens 6 Stunden pro Woche persönlich in einem Wirtschaftszweig tätig sind; ohne Arbeitslose. InTeilzeit erwerbstätige Personen: Personen, die während weniger als den in der Branche oder im Betrieb üblichen Arbeitsstunden beschäftigt sind. Erfassung am Wohnort, über die Pendlerstatistik auch für die Arbeitsgemeinde; 1980 erstmals auch nach Wirtschaftszweigen.

Beschäftigte (Betriebszählung): In der betreffenden Arbeitsstätte beschäftigte Personen, inkl. Inhaber, mitarbeitende Familienangehörige. Unterscheidung in Personen mit 30 und mehr Wochenstunden und Personen mit weniger als 30 Wochenstunden Arbeitszeit (1975) bzw. Beschäftigte mit mindestens $70 \%$ und Beschäftigte mit weniger als $70 \%$ der betriebsüblichen Arbeitszeit für Vollzeitbeschäftigte (1985).

Tertiärer Sektor: Da sich der größere Teil der Zahlen auf das Jahr 1980 bezieht (Volkszählung), wird von der damals verwendeten Wirtschaftssystematik ausgegangen (BUNDESAMT FÜR STATISTIK, 1981). In Abweichung davon wurden in einzelnen Zählungen folgende größere Bereiche nicht bzw. zusätzlich zum tertiären Sektor gezählt:

- 1960: nicht beim tertiären Sektor gezählt: Reparaturgewerbe, Architektur- und Bauingenieurbüros;

- 1970: nicht beim tertiären Sektor gezählt: Reparaturgewerbe, Architektur- und Bauingenieurbüros; zusätzlich beim tertiären Sektor gezählt: Kehrichtbeseitigung, Umweltschutz;

- 1975: keine größeren Verschiebungen

- 1985: zusätzlich beim tertiären Sektor: Kehrichtbeseitigung, Umweltschutz.

Ernst K. Gächter, Dr., Amt für Statistik der Stadt Bern, Gutenbergstrasse 27, 3001 Bern 
Da entsprechende Detailangaben z. T. fehlen, sind diese Verschiebungen in den in den Tabellen enthaltenen Zahlen nicht berücksichtigt, d. h. ein Teil des Zuwachses des tertiären Sektors zwischen 1970 und 1980 z. B. ist auf das neu dazugezählte Reparaturgewerbe und die Architektur- und Bauingenieurbüros zurückzuführen.

Die offiziellen Strukturzahlen nach Wirtschaftssektoren beruhen auf der institutionellen Betrachtungsweise (siehe HOTZ/WUERTH, 1985). So zählt z.B. die Forschungsabteilung einer industriellen Großfirma mit hohem Akademikeranteil zum sekundären Sektor, während der Gärtner eines Hotels als Beschäftigter des tertiären Sektors erfaßt wird. Die Zahlen nach Sektoren müssen deshalb in diesem Sinne bewertet werden.

Öffentliche Verwaltung: Umfaßt die folgenden Bereiche: allgemeine öffentliche Verwaltung, Polizei, Feuerwehr, Rechtspflege, Strafvollzugsanstalten, Landesverteidigung, Sozialversicherung (SUVA, AHV/IV, ALV; 1980 unter «Banken, Versicherungen»). Nicht enthalten sind darin aber die Generalund Kreisdirektionen von SBB und PTT sowie die städtischen Verkehrs- und industriellen Betriebe.

Arbeitgeber öffentliche Hand: Beschäftigte in Verwaltungen und Betrieben der öffentlichen Wirtschaft, nämlich der Eidgenossenschaft (inkl. SBB, PTT), der Kantone (inkl. Bezirke), der Gemeinden (inkl. Bürger-, Schul- und Kirchgemeinden), der ausländischen Staaten (Botschaften, Konsulate), der internationalen Organisationen (in Bern: Weltpostverein und Internationales Eisenbahnamt) und anderer öffentlich-rechtlicher Körperschaften (Genossenschaften, Stiftungen).

Beamte: Der Begriff des Beamten ist nirgends allgemeinverbindlich definiert, sondern wird von Fall zu Fall unterschiedlich angewendet. So kennt das Bundesamt für Statistik bei den erlernten/ausgeübten Berufen die Begriffe «Leitende Beamte der öffentlichen Verwaltung», "Stationsbeamte» bei den Bahnen, «Schalterbeamte» bei der Post u. a., reiht aber das Verwaltungspersonal unter den Büroberufen (Kaufmännische Angestellte, Buchhalter usw.) ein. Bei der beruflichen Stellung wird unterschieden in leitende und untere Angestellte, gelernte und angelernte Arbeiter usw., während der Begriff des «Beamten» nicht vorkommt. Die Personalstatistik der Stadt Bern kennt folgende Kategorien von Gemeindepersonal: Beamte, Arbeiter, Heim- und Pflegepersonal, Lehrer, u. a. Der Begriff des Beamten ist dabei vor allem anstellungsrechtlich definiert. $\mathrm{Zu}$ den «Beamten» zählen das Verwaltungspersonal und die Polizeibeamten; zu den «Arbeitern» gehören u. a. die Fahrdienstangestellten der Verkehrsbetriebe, Teile der Beschäftigten der Industriellen Betriebe und des Straßeninspektorates.

\section{Entwicklung der Beschäftigten des tertiären Sektors und der öffentlichen Verwaltung in Bern}

Wenn wir die Erwerbstätigen mit Wohnort Bern betrachten (Tabelle 1), so hat sich der Anteil der im tertiären Sektor Beschäftigten (ohne Berücksichtigung der Änderungen in der Sektorenzuteilung) von $60 \%$ im Jahre 1960 über $64 \%$ im Jahre 1970 auf $71 \%$ im Jahre 1980 erhöht. Die absolute Zahl der Beschäftigten insgesamt und in geringerem Maße der Beschäftigten im tertiären Sektor ist aber wegen des Bevölkerungsverlustes der Stadt Bern seit 1970 zurückgegangen. Ein Vergleich der Beschäftigten in der öffentlichen Verwaltung mit dem Total der Beschäftigten mit Wohnort Bern ergibt für 1960 einen Anteil von 8,8\%, 1970 9,4\% und 1980 9,7\%. Dieser Anteil hat sich somit in den zwanzig Jahren leicht erhöht. Nehmen wir aber die öffentliche Hand insgesamt als Arbeitgeber, so stieg der Anteil am Total der Beschäftigten mit Wohnort Bern von $25,4 \%$ 1960 über 27,4\% 1970 auf 30,4\% im Jahre 1980 an.

Für die Beschäftigten mit Arbeitsort Bern stehen uns Vergleichszahlen für die Jahre 1975 (Betriebszählung), 1980 (Volkszählung, Erwerbstätige nach Arbeitsort) und 1985 (Betriebszählung) zur Verfügung. Der Anteil des tertiären Sektors (Vollzeiterwerbstätige bzw. Beschäftigte mit 30 und mehr Wochenstunden) erhöhte sich von $68,2 \% 1975$ über $70,7 \% 1980$ auf $73,7 \%$ 1985. Die entsprechenden Zahlen für die öffentliche Verwaltung lauten $12,2 \%$, $12,4 \%$ und $13,0 \%$. Wenn wir die öffentliche Hand als Arbeitgeber betrachten, so veränderte sich der Anteil an den Beschäftigten in Bern insgesamt von 31,6\% 1975 über 33,8\% 1980 auf 33,7\% 1985 .

Nach der Beschäftigtenzahl dominierten in Bern 1985 im tertiären Sektor die folgenden Wirtschaftsklassen: Öffentliche Verwaltung (14 400), Detailhandel (9900), Gesundheitswesen (9400), Nachrichtenübermittlung (6800), Beratung/Planung (6200), Gastgewerbe (4800) und Unterrichtswesen (4200 Beschäftigte mit mindestens $70 \%$ der betriebsüblichen Arbeitszeit). Auf die restlichen Wirtschaftsklassen des tertiären Sektors entfielen noch rund 25700 Beschäftigte.

Interessant ist die Aufteilung der Beschäftigten bei der öffentlichen Hand nach den verschiedenen Wirtschaftszweigen und ihr Anteil am Total der jeweiligen Beschäftigten des betreffenden Wirtschaftszweiges (Tabelle 2). Zum Verständnis der Tabelle sind noch einige Bemerkungen nötig: Beim primären Sektor handelt es sich vorwiegend um Gärtnereien (Stadtund Bundesgärtnerei), bei der Industrie macht die Waffenfabrik (Bund) einen wesentlichen Teil aus. Zur Wirtschaftsabteilung «Handel, Banken, Versicherungen usw.» zählen die Kantonalbank, die Gebäudeversicherung (Kanton) und 1980 auch die Sozialversicherung (Bund, Kanton, Stadt). Beim Reise- und Transportgewerbe entfallen rund $80 \%$ 
Stadt Bern: Erwerbstätige / Beschāftigte im tertiärem Sektor und bei der öffentlichen Hand 1960 - 1985

\begin{tabular}{|c|c|c|c|c|c|c|c|c|}
\hline \multirow[t]{2}{*}{ Merkmale } & \multicolumn{3}{|c|}{ Erwerbstätige mit Wohnort Bern } & \multicolumn{5}{|c|}{ Beschäftigte mit Arbeitsort Bern } \\
\hline & VZ 1960 & VZ 1970 & VZ 1980 & BZ 1975 & vz 1 & 1980 & $\mathrm{BZ}$ & 1985 \\
\hline \multicolumn{9}{|l|}{ Erwerbstätige / Beschäftigte insgesamt: } \\
\hline alle Beschäftigten & 80412 & 83742 & 72409 & 122418 & 125 & 5114 & 135 & 082 \\
\hline davon Vollbeschäftigte & 78168 & 72889 & 60664 & 107508 & 107 & 7006 & 110 & 379 \\
\hline \multicolumn{9}{|l|}{ Wirtschaftszweig: } \\
\hline alle Beschäftigten & $48 \quad 247$ & $53 \quad 547$ & 51209 & 86311 & 90 & 623 & & * \\
\hline davon Vollbeschäftigte & 46552 & $45 \quad 277$ & 41575 & 73295 & 75 & 5618 & 81 & 348 \\
\hline \multicolumn{9}{|l|}{ Oeffentliche Verwaltung: } \\
\hline alle Beschăftigten & 7115 & 7873 & 6995 & 13867 & 14 & 4566 & & * \\
\hline davon Vollbeschäftigte & * & 7116 & 6138 & 13109 & 13 & 3261 & 14 & 387 \\
\hline \multicolumn{9}{|l|}{ Arbeitgeber $^{1)}$ : } \\
\hline \multicolumn{9}{|l|}{ Oeffentliche Hand insgesamt: } \\
\hline alle Beschäftigten & 20390 & 22954 & 21978 & * & 40 & 995 & & * \\
\hline davon Vollbeschäftigte & 20106 & 20670 & 18945 & 33922 & 36 & 5220 & 37 & 233 \\
\hline \multicolumn{9}{|l|}{ Stadt Bern, andere Gemeinden: } \\
\hline alle Beschäftigten & 4912 & 5607 & 5911 & * & 8 & 3055 & & * \\
\hline davon Vollbeschäftigte & 4804 & 4998 & 4899 & 5527 & & 5674 & 5 & 317 \\
\hline \multicolumn{9}{|l|}{ Kanton Bern (inkl. Bezirke): } \\
\hline alle Beschäftigten & 3624 & 5293 & 6184 & * & 11 & 1390 & & * \\
\hline davon vollbeschäftigte & 3575 & 4644 & 5119 & 8850 & & 9557 & 7 & 258 \\
\hline \multicolumn{9}{|l|}{ Eidgenossenschaft (inkl. SBB, PTT): } \\
\hline alle Beschäftigten & 11353 & 11323 & 9266 & * & 20 & 668 & & * \\
\hline davon vollbeschäftigte & 11238 & 10346 & 8349 & 19044 & 19 & 9166 & 19 & 784 \\
\hline \multicolumn{9}{|l|}{ Ausländische Staaten, internat. Organisationen: } \\
\hline alle Beschãftigten & 501 & 731 & 617 & . & & 882 & & . \\
\hline davon Vollbeschäftigte & 489 & 682 & 578 & . & & 823 & & \\
\hline \multicolumn{9}{|l|}{ Andere öffentl.-rechtl. Körperschaft ${ }^{2)}:$} \\
\hline alle Beschäftigten & . & & . & * & & . & & * \\
\hline davon Vollbeschäftigte & . & . & . & 501 & & . & & 874 \\
\hline
\end{tabular}

1) Betriebszählungen: ohne primären Sektor (siehe Tabelle 2).

2) In der Volkszählung werden die anderen öffentlich-rechtlichen Körperschaften der entsprechenden Stufe zugeordnet (d.h. Burger- und Kirchgemeinden zu "Gemeinde", Inselspital-Stiftung zu "Kanton" usw.). In der Betriebszählung 1975 sind die anderen öffentl.-rechtl. Körperschaften, 1985 auch die Stiftungen und Genossenschaften offentlichen Rechts separat er- 
auf die SBB (Betrieb und Verwaltung), der Rest auf die städtischen Verkehrsbetriebe. Das Unterrichtswesen teilt sich etwa hälftig auf Stadt Bern (Primarund Sekundarschule, Mittelschulen, Berufsschulen) und Kanton Bern (Hochschule) auf. Beim Gesundheitswesen dominiert der Kanton mit rund
$80 \%$, der Rest entfällt vorwiegend auf die beiden Regionalspitäler (früher Stadtspitäler). Bei den zusammengefaßten übrigen Wirtschaftszweigen spielen Museen und Bibliotheken noch eine wichtigere Rolle. Leider waren hier die Zahlen für 1985 noch nicht verfügbar.

Tab. 2

Stadt Bern: Beschäftigte bei der öffentlichen Hand nach Wirtschaftszweigen 1975, 1980 Wirtschaftszweig

Beschäftigte am Arbeitsort Bern
BZ $1975^{1)}$
VZ $1980^{2)}$

absolut in ${ }^{3)}$ absolut in $\%^{3)}$

Beschäftigte bei der öffentlichen Hand insgesamt

33922

31,7

40995

32,8

Primärer Sektor (vorw. Gartenbau)

Industrie, Handwerk

Baugewerbe

Energiewirtschaft, Umweltschutz

Handel, Banken, Versicherungen usw.

Reise-, Transportgewerbe

Post, Fernmeldebetriebe, Nachrichtenübermittlung

Oeffentliche Verwaltung

Ausländische diplomatische Vertretungen, internationale Organisationen

Unterrichtswesen, Wissenschaft

Gesundheitswesen

Wohlfahrtspflege, soziale Hilfswerke

Kirchliche Organisationen

Uebrige Wirtschaftszweige

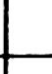


Tab. 3

Städtevergleich: Erwerbstătige / Beschäftigte im tertiären Sektor und bei der öffentlichen Hand 1980/1985

\begin{tabular}{|c|c|c|c|c|c|c|c|c|c|c|c|c|}
\hline Stadt & insgesamt & \multicolumn{7}{|c|}{ 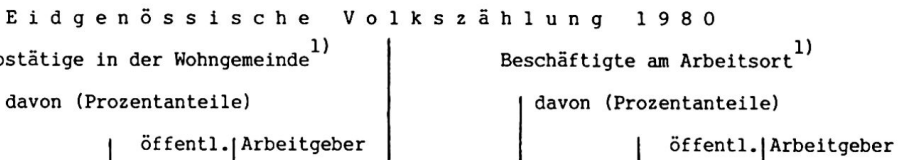 } & \multicolumn{4}{|c|}{$\begin{array}{c}\text { Eidgenössische Betriebszählung } 1985 \\
\text { Beschäftigte am Arbeitsort } \\
\text { () }^{\text {davon (Prozentanteile) }}\end{array}$} \\
\hline Bern & 72409 & 70,7 & 10,5 & 30,4 & 125114 & 72,4 & 12,3 & 32,8 & 110379 & 73,7 & 12,4 & 33,7 \\
\hline Zürich & 193958 & 71,3 & 3,5 & 19,3 & 305957 & 71,8 & 3,2 & 17,3 & 273672 & 74,5 & 3,2 & 16,8 \\
\hline Basel & 91668 & 61,5 & 3,1 & 18,9 & $152500^{6)}$ & $60,0^{6)}$ & 3,0 & 18,0 & 130417 & 62,6 & 3,0 & 16,3 \\
\hline Biel & 27235 & 48,8 & 3,2 & 17,2 & 36359 & 52,0 & 2,7 & 17,8 & 30123 & 55,2 & 2,8 & 19,7 \\
\hline Schweiz total & $\begin{array}{ll}3067233\end{array}$ & 53,8 & 3,7 & 16,1 & $3164400^{6)}$ & $53,1^{6)}$ & 3,6 & 15,6 & 2961378 & 54,8 & 3,3 & 14,5 \\
\hline
\end{tabular}

1) Alle Erwerbstätigen (Voll- und Teilzeitbeschäftigte).

2) Beschäftigte mit mindestens 70 \& der betriebsüblichen Arbeitszeit für Vollbeschäftigte.

3) Inklusive ausländischen diplomatischen Vertretungen, internationale Aemter und Organisationen.

4) Ohne Sozialversicherung.

5) Ohne Beschäftigte der öffentlichen Hand im primären Sektor.

6) Inklusive geschätzte Zahlen für Grenzgänger. 


\section{Die Situation in Bern im Vergleich mit anderen Schweizer Städten}

Um die Frage nach dem Gewicht des tertiären Sektors und der öffentlichen Verwaltung in der Stadt Bern beantworten zu können, wollen wir noch Vergleiche mit anderen Städten und der Schweiz insgesamt vornehmen (Tabelle 3 ). Wir beschränken uns auf die beiden anderen Deutschschweizer Großstädte (Zürich, Basel) und die beiden größeren Nachbarstädte im Kanton Bern (Biel, Thun). Für den Vergleich ziehen wir Zahlen aus der Volkszählung 1980 (Erwerbstätige am Wohnort, am Arbeitsort) und der Betriebszählung 1985 bei.

Bei den wohnhaften Erwerbstätigen ist der Anteil des tertiären Sektors in Bern annähernd gleich groß wie in Zürich, während Basel deutlich tiefer liegt. Thun hat einen Wert um den gesamtschweizerischen Durchschnitt, während Biel deutlich darunter liegt. Ein großer Unterschied ergibt sich aber beim Anteil der öffentlichen Verwaltung, der in Bern rund das Dreifache der Werte von Zürich, Basel, Biel oder der Schweiz insgesamt ausmacht. Eine Mittelstellung nimmt Thun ein (Waffenplatz). Auch der Anteil des Arbeitgebers öffentliche Hand an den Beschäftigten ist in Bern deutlich höher gegenüber Zürich, Basel oder der Schweiz insgesamt. Für die $B e$ schäftigten am Arbeitsort mußten bei Basel und der Schweiz insgesamt zu den Daten der Volkszählung noch die Grenzgänger zuaddiert werden. Die Zahlen für 1980 stimmen in der Größenordnung bei den betrachteten Städten mit denjenigen nach dem Wohnort überein (Anteile am Arbeitsort gleich oder eher etwas tiefer), bei der Stadt Bern dagegen ergibt sich ein umgekehrtes Bild: Die Anteile des tertiären Sektors, der öffentlichen Verwaltung und des Arbeitgebers öffentliche Hand sind bei den Beschäftigten in der Stadt Bern deutlich höher als bei den wohnhaften Erwerbstätigen. D. h. die betreffenden Personenkategorien wohnen im Vergleich in höherem Maße außerhalb der Stadt Bern und sind damit Zupendler. Als Beispiel sei der Prozentanteil der Vollzeit-Beschäftigten in der Stadt Bern 1980 mit Wohnort Bern angeführt: Insgesamt $51,2 \%$; Arbeitgeber: Privatwirtschaft 51,9\%, öffentliche Hand zusammen $49,8 \%$, Stadt Bern $69,6 \%$, Kanton Bern $51,5 \%$, Eidgenossenschaft $42,3 \%$.

Die Zahlen aus der Betriebszählung 1985 (Beschäftigte mit mindestens $70 \%$ der betriebsüblichen Arbeitszeit; Volkszählung: Voll- und in Teilzeit Beschäftigte) deuten auf eine weitere Zunahme des Anteils der öffentlichen Verwaltung in der Stadt Bern hin. Da die Teilzeitbeschäftigten unterschiedlich stark in den einzelnen Wirtschaftszweigen vertreten sind (hohe Anteile z. B. im Detailhandel, im Unterrichtswesen) und ihre Zahl vermutlich überproportional zugenommen hat, sind Vergleiche nur bedingt möglich.
Zusammengefaßt kann gesagt werden, daß die öffentliche Verwaltung in der Stadt Bern ein deutlich höheres Gewicht hat als in anderen Städten, zumal wenn wir noch die Beschäftigten bei der Generaldirektion und den zentralen Diensten von SBB und PTT dazurechnen (5200 Beschäftigte 1975; THOMET, 1975). Die öffentliche Verwaltung in diesem weiteren Sinne umfaßte 1980 rund $16 \%$ aller Beschäftigten in der Stadt Bern.

Ob damit Bern zu recht als «Beamtenstadt» qualifiziert wird, bleibe dahingestellt, zumal der Begriff des Beamten nicht allgemeingültig definiert ist. Im weiteren kann vermutet werden, daß nicht nur die Zahl, sondern auch die angeblich spezielle Mentalität des Beamten zu dieser Klassifizierung geführt haben.

\section{Auswirkungen der Verwaltungskonzentration in Bern auf die Stadtstruktur}

Die festgestellte Konzentration von Arbeitsplätzen der öffentlichen Verwaltung und der öffentlichen Hand allgemein in der Stadt Bern hat Auswirkungen auf die Bevölkerungs- und Wirtschaftsstruktur sowie die finanzielle Situation der Stadt, welche aber im Rahmen dieses Artikels nur summarisch dargestellt werden können. Vielfach sind diese Einflüsse auch nicht oder nur schwer quantifizierbar. Zudem muß offenbleiben, was privatwirtschaftliche Arbeitsplätze an Stelle solcher der öffentlichen Hand der Stadt an Kosten/Nutzen bringen würden. Die hohe Zahl von relativ sicheren und konjunkturunabhängigen Arbeitsplätzen in der öffentlichen Verwaltung von eher überdurchschnittlicher Qualifikation und Entlöhnung bildet für die Stadt Bern ein positives Element, das aber nur schwer quantifizierbar ist (Steuereinnahmen, Krisensicherheit). Da aber gerade das Personal der Eidgenossenschaft eine hohe Pendlerquote aufweist (siehe weiter unten), wird dieser Vorteil relativiert.

Im weiteren haben Bund und Kanton als Einkäufer und Auftraggeber Auswirkungen auf die stadtbernische Industrie, das Gewerbe und den Handel. Diesbezügliche Detailuntersuchungen liegen aber nur im kantonalen Rahmen vor (diverse Arbeiten im Rahmen des Nationalen Forschungsprogrammes Nr. 5 «Regionalprobleme»). Auch das Gastgewerbe profitiert von der Stellung der Stadt Bern als Bundeshauptstadt («Parlamentariertourismus», Werbeeffekt der Bundesstadt). Sofern durch die Parlamentarier oder Bundesbeamten aber Zweitwohnungen (ohne Steuerdomizil) in der Stadt Bern belegt werden, ergibt sich ein negativer Effekt (Verdrängung von Bewohnern mit Erstwohnsitz und Steuerdomizil in Bern).

Belastungen können für die Stadt Bern aus der Verwaltungskonzentration durch direkte Kosten oder 
durch Einnahmenausfälle entstehen. Direkte Kosten erwachsen der Stadt Bern vorwiegend in den Bereichen Sicherheit (polizeilicher Ordnungsdienst, Schutzbewachungen u. ä.; z. T. vom Bund abgegolten) sowie privater und öffentlicherVerkehr (Konzentration der Arbeitsplätze der öffentlichen Verwaltung in City- und Cityrandlagen).

Einnahmenausfälle entstehen der Stadt Bern durch die öffentlichen Verwaltungen (Kanton, Bund, SBB, PTT) und die diplomatischen Vertretungen in folgenden Gebieten: Die öffentliche Hand als Arbeitgeber bezahlt keine Steuern. Zudem ist bei den Botschaften und internationalen Organisationen zum Teil auch das Personal von verschiedenen Steuern befreit. Im weiteren werfen die Liegenschaften der öffentlichen Verwaltung keine Liegenschaftssteuern ab. Der Steuerausfall bei der Liegenschaftssteuer (Bund, Generaldirektion PTTund SBB, Botschaften, Weltpostverein) wurde 1974 auf $400000 \mathrm{Fr}$. und derjenige der Einkommenssteuer bei den Botschaftsangestellten auf 6,25 Mio Fr. oder knapp 4\% der eingegangenen Einkommenssteuer geschätzt (THOMET, 1975). Die Steuereinnahmen bei privatwirtschaftlicher Nutzung der von der öffentlichen Verwaltung belegten Nutzflächen (Kapital- und Gewinnsteuern von juristischen Personen z. B.) würden von der Art der Nutzung abhängen und lassen sich damit nur schwer quantifizieren. Immerhin kann als Indiz angefügt werden, daß in Bern der Anteil der juristischen Personen (Kapital- und Gewinnsteuern) am gesamten Steuerertrag 1984 nur $12 \%$ betrug, während der Wert für Zürich bei $28 \%$ lag.

Ein (mindestens theoretischer) Steuerentgang entsteht der Stadt Bern auch bei den Beschäftigten, die außerhalb der Stadt wohnen. Verschärft wird dieser Ausfall noch durch den Umstand, daß das Bundespersonal in überdurchschnittlichem Ausmaße (1980: 57,7\% Zupendler), das keine Steuern zahlende Botschaftspersonal dagegen nur in stark unterdurchschnittlichem Maße (23\%; THOMET, 1975) unter den Zupendlern zu finden ist (vom Total aller Beschäftigten 1980 in der Stadt Bern waren 48,8\% Zupendler).

Der Entgang von Einkommenssteuern aus dem Arbeitseinkommen der auswärts wohnhaften Angestellten der Bundesverwaltung (inkl. Generaldirektion und zentrale Dienste von SBB und PTT) kann grob auf 30 Mio Fr. 1980 geschätzt werden $(8000$ vollbeschäftigte Zupendler, durchschnittliches
Einkommen 50000 Fr., Gemeindesteuerbetrag 3700 Fr.). Durch den Raumanspruch der Verwaltung (Bund, Kanton, SBB, PTT) entstehen auch Auswirkungen auf die Boden- und Liegenschaftspreise und die Zweckentfremdung/Verdrängung von Wohnraum (siehe THOMET, 1975).

Abschließend kann festgestellt werden, daß die Verwaltungskonzentration in Bern positive und negative Einflüsse auf die Bevölkerungs-, Wirtschaftsund Finanzstruktur der Stadt Bern hat. Eine eindeutige Bilanz, vor allem quantitativer Art, kann nicht gezogen werden.

\section{Literatur- und Quellenverzeichnis}

\section{Literatur}

Amt für Statistik der Stadt Bern (1986): Die Verflechtungen der Kernstadt Bern mit ihrem Umland, insbesondere mit den Partnergemeinden der Region Bern, Teil A: Externe Effekte durch Bereitstellung der Infrastruktur. In: Berner Beiträge zur Stadt- und Regionalforschung, Mai 1986.

BUCHER, J. (1975): Studie über den Arbeitsmarkt in Stadt und Region Bern. In: Berner Beiträge zur Stadt- und Regionalforschung, Heft 2.

Bundesamt für Statistik (1981): Eidgenössische Volkszählung 1980. Systematik der Wirtschaftszweige, Arbeitsdokumente für die schweizerische Statistik, Heft 6d.

GÄCHTER, E. (1978): Untersuchungen zur kleinräumigen Bevölkerungs-, Wohn- und Arbeitsplatzstruktur der Stadt Bern. In: Geographica Helvetica, 33. Jg., 1, Seite 1-16.

GÄCHTER, E. (1980): Kosten und Benutzung städtischer Infrastrukturen am Beispiel von Bern. In: Geographica Helvetica, 35. Jg., 4, Seite 170-178.

HOTZ-HART, B., WÜRTH, M. (1985): Strukturwandel im Dienstleistungssektor und Stadtentwicklung. In: DISP 80/81, Seite 17-23.

THOMET, R. (1975): Die wirtschaftliche Belastung der Stadt Bern als Bundessitz, schriftliche Arbeit Rechts- und wirtschaftswissenschaftliche Fakultät Universität Bern. In: Stadt- und Regionalforschungsstelle Bern, Graue Heftreihe, Nr. 49.

\section{Statistische Quellen}

Auswertungen (z.T. unveröffentlichte Manuskripttabellen) der Eidg. Volkszählungen 1960, 1970, 1980 und der Eidg. Betriebszählungen 1975 und 1985, deponiert im Amt für Statistik der Stadt Bern.

Der Autor dankt an dieser Stelle dem Bundesamt für Statistik (Herren Geißelhardt und Wernli, Sektion Volkszählung, und Herr Poffet, Sektion Unternehmungsstatistik) für die zur Verfügung gestellten Unterlagen. 